\title{
CRISIS Y FUTURO DE LA INGENIERÍA
}

\author{
Darío Valencia Restrepo*
}

"La mejor manera de predecir el futuro es construirlo".

PeterF. Drucker

\section{RESUMEN}

El autor del artículo sostiene que la ingeniería vive en la actualidad una crisis que se manifiesta no sólo en Colombia sino en el ámbito internacional, y que para salir de ella se requiere preparar un nuevo tipo de ingeniero. Se señalan los atributos que debe poseer dicho profesional, además de otros que le han sido tradicionales. Se discuten los

S.M. \& C.E. del Instituto Tecnológico de Massachusetts.

Profesor Emérito y Profesor Titular de Ingeniería Civil en la Universidad Nacional de Colombia. paradigmas que se han dado en la historia de la ingeniería, con el fin de proponer un nuevo paradigma para el futuro. Finalmente, se incluyen algunos comentarios sobre el impacto de la era digital e Internet. 


\section{ABSTRACT}

The author points out that the engineering profession is undergoing a significant crisis not only in Colombia but also at the international level. A new engineer with certain attibutes is required if the profession wants to have a leading role in society. Three paradigms in engineering history are described, followed by the suggestion of a new paradigm for the future. Finally, some brief comments are presented about the impact of Internet and the digital era on engineering education, and about the challenges Colombia faces in this respect.

\section{INTRODUCCION}

Tanto en Colombia como en el ámbito internacional existe un cierto consenso sobre la existencia de una crisis que afecta a la ingeniería tradicional. Es un hecho que la profesión ha perdido presencia y liderazgo en los asuntos relacionados con el desarrollo social y material de los países, al tiempo que se echa de menos los extraordinarios aportes de la ingeniería en las primeras décadas y mediados del siglo $X X$. ¿Cuáles son los elementos y manifestaciones de esa crisis? ¿Qué nuevos atributos, amén de los tradicionales, exigen a la profesión los nuevos tiempos? ¿Cuáles cambios en la formación de los estudiantes deben contemplar las facultades de ingeniería?

\section{ELEMENTOS DE LA CRISIS}

La concentración del ingeniero en lo técnico y el orgullo de su técnica le han impedido a ese profesional la visión sistémica, lo han llevado a desconocer con frecuencia implicaciones sociales, y le han dificultado el diálogo con otras disciplinas y profesiones. Su poca relación con las humanidades y el arte explica en algún grado una pérdida del sentido de grandeza, sentido que se relaciona con los aspectos éticos y estéticos que debe llevar consigo toda obra de ingeniería.

En el caso colombiano se agregan, además, algunos elementos específicos: ausencia de investigación y de estímulo a la creatividad y a la innovación; poca atención a graves problemas nacionales; al igual que en otros sectores de la vida nacional, la corrupción ha penetrado el ejercicio de la ingeniería; el peso del individualismo y la falta de solidaridad se oponen al trabajo en grupo y a las construcciones colectivas; y la proliferación de carreras y denominaciones están llevando a una seria disminución de calidad y a una pérdida de identidad en la profesión. A propósito, según datos recientes, había en el país 398 programas de ingeniería con 69 denominaciones distintas, y una de éstas era iingeniería de radio y televisión!

La poca relación de los grupos universitarios de investigación con la empresa y los centros de desarrollo tecnológico, el retraso en la formación doctoral en ingeniería, y el predominio de la adquisición o adaptación de tecnologías del exterior, son a la vez causa y efecto de la poca investigación que se lleva a cabo en las Facultades de Ingeniería. Con respecto al desarrollo tecnológico, existen tres categorías de países: los que lo producen, los que los transfieren y adaptan, $y$, finalmente, aquellos que lo compran ciegamente. Cabe preguntarse en qué medida Colombia está cada vez más en la tercera categoría.

Da pesar observar con frecuencia la poca o nula transferencia tecnológica $o$ de conocimientos que el país obtiene cuando se emprenden grandes proyectos de infraestructura por parte de firmas del exterior, 0 cuando se adquieren patentes y licencias provenientes de países desarrollados. Señala un estudio reciente de la Universidad de los Andes y la Corporación para la Investigación y la Docencia Económica, de Medellín (1), que en el medio industrial es clara la ausencia de flujos nacionales de conocimientos 
tecnológicos y la pobreza de ingenieros con altos niveles de formación. En pocas palabras, que es marginal el papel de la capacidad científica nacional en la rápida transformación tecnológica que el sector productivo ha experimentado en los últimos años.

\section{MANIFESTACIONES DE LA CRISIS}

Múltiples son las expresiones de la crisis y entre ellas podría citarse la pérdida de aprecio social por la ingeniería, la ausencia de liderazgo de la profesión, la casi inexistencia de comunidades técnicas, el marchitamiento de las sociedades profesionales, el desempleo y subempleo que padecen los ingenieros, y la constante pérdida de cargos técnicos del sector público que tradicionalmente habían sido ocupados por profesionales de la ingeniería.

El pasado enseña que los ingenieros ocuparon un papel preponderante en la sociedad cuando se vincularon al desarrollo industrial y se preocuparon por los efectos de la acción profesional sobre aspectos sociales y administrativos. El proyecto de la Escuela de Minas, emprendido por don Tulio Ospina y otros pioneros hacia fines del siglo XIX, fue exitoso porque entendió la función que la ciencia y la tecnología podían cumplir en la construcción de un país moderno. Hoy las circunstancias y los medios son bien distintos pero la finalidad tiene cierta analogía: cómo diseñar una ingeniería que responda a la necesidad de crear una nueva sociedad en un país agobiado por la violencia y la intolerancia, retrasado en el conocimiento científico y tecnológico, y cada vez más ajeno a las condiciones de competencia que reclaman los nuevos escenarios de la economía internacional.

Además del desempleo estructural que afecta a la profesión, ha aparecido el fenómeno del subempleo, sobre todo cuando los ingenieros desempeñan tareas que podría realizar un tecnólogo. Aquí existen más ingenieros que tecnólogos, al revés de lo que ocurre en el medio internacional, al parecer por una subestimación del papel que pueden cumplir aquellos últimos. Por ello es útil señalar que un libro titulado "La ventaja competitiva de las naciones", publicado en 1990 y cuyo autor es Michael E. Porter, puso de presente la significativa contribución de las instituciones técnicas de Alemania e Italia a los aumentos de la productividad en dichos países.

Sobre la pérdida de cargos tradicionales, es pertinente preguntarse en qué medida ello se explica por el frecuente desentendimiento del ingeniero con respecto a aspectos políticos y sociales.

\section{ATRIBUTOS DE UN NUEVO INGENIERO}

La crisis de la ingeniería se ha acentuado en razón de una serie de factores aparecidos en las últimas décadas, en especial relacionados con la complejidad de los problemas, así como con la existencia de múltiples intereses y actores. La dimensión técnica de un asunto dado sigue siendo importante, pero además es necesario lidiar con grupos de presión, interactuar con ambientalistas, tener en cuenta aspectos políticos, sociales y legales y, en general, entenderse con un nuevo ciudadano más consciente y existente que en el pasado. De otro lado, la era del conocimiento y la información, así como la velocidad del cambio tecnológico, están transformando la sociedad y la industria, y a la vez afectando el ejercicio profesional de la ingeniería.

Siguen teniendo vigencia ciertas cualidades tradicionales del ingeniero como apego a la realidad, sentido de lo cuantitativo, capacidad de modelar, servir de puente entre la ciencia y la tecnología, potencial como innovador y líder para la industria. Pero ellas ya 
no son suficientes, y es necesario considerar otros atributos atinentes a la orientación del uso de la tecnología, la capacidad interdisciplinaria, el buen uso del lenguaje y la comunicación, la percepción de las relaciones entre lo técnico, administrativo, político, económico, ambiental...

En suma, se requiere un profesional de la síntesis y la integración, o sea, un profesional que sea capaz, además de separar para analizar, de reunir para sintetizar o complejificar, de modo que un problema dado aparezca en su contexto natural. La visión reduccionista, aquella que se concentra exclusivamente en la tarea aislada, destruye la solidaridad y la responsabilidad. Podría decirse, entonces, que el pensamiento sintético o complejo lleva consigo una misión ética.

Lo anterior exige un trabajo interdisciplinario en el cual el ingeniero puede desempeñar una tarea muy significativa si es capaz de sostener un diálogo respetuoso con otros saberes, y al mismo aporta, en especial, su capacidad de buscar soluciones óptimas o cercanas al óptimo no sólo en lo técnico económico sino también en lo político, social, ambiental...

\section{PARADIGMAS EN LA HISTORIA DE LA INGENIERÍA}

Tres paradigmas se han dado en la historia de la ingeniería. Hasta fines del siglo XVIII no existía la profesión tal como se conoce hoy; se construía intuitivamente, con base en ensayo y error, y a la manera de los artesanos según la tradición de maestros y aprendices. Podría decirse que los embriones de arquitecto e ingeniero se confundían en el Maestro Constructor, y ésta trabajaba en el sitio de la obra. Éste es el primer paradigma.

Debido a las exigencias de la revolución industrial, y gracias a la aparición de las primeras escuelas de ingeniería en Francia, empieza a perfilarse la ingeniería como arte (segundo paradigma), es decir, como un oficio especializado que exigía destrezas y habilidades muy elaboradas.

El segundo paradigma dura más o menos hasta la segunda guerra mundial. Luego aparece la ingeniería con base científica, el tercer paradigma. Se aprovechan al máximo las ciencias exactas y naturales para fundamentar la profesión, se desarrollan las llamadas ciencias de la ingeniería como la hidráulica, la resistencia de los materiales, las estructuras, etc., y se acelera la creación de nuevas ramas de la ingeniería, distintas a la civil.

La necesidad de sintetizar e integrar las diferentes miradas o dimensiones de un problema llevan a proponer un nuevo paradigma para la ingeniería, acorde con los tiempos que corren, y que podría llamarse el de Maestro Integrador. Este cuarto paradigma tiene cierta coincidencia con ideas expresadas en artículos y seminarios realizados en Colombia durante la última década (2) (en especial la ponencia "La ingeniería y su impacto social y económico"), y también recoge conceptos emitidos en un importante coloquio internacional sobre el futuro de la Ingeniería Civil y Ambiental, llevado a cabo hacia principios del año 2000 en el Instituto Tecnológico de Massachusetts (3).

\section{UNA TAREA PARA LAS FACULTADES DE INGENIERÍA}

La obtención de los atributos profesionales antes señalados exige cambios académicos a las Facultades de Ingeniería, algunos de ellos de carácter radical. Dichos cambios deben ser estudiados por directivas, profesores, estudiantes, pero hoy más que nunca se requiere una urgente participación de esa discusión por parte de sociedades profesionales y egresados, consultores, empresarios, gobernantes y políticos.

Un primer punto se refiere a la ineficacia de la 
docencia tradicional y de la acumulación de cursos en las carreras de ingeniería. Es imperativo aprovechar nuevos escenarios 0 encuentros pedagógicos, como los relacionados con los seminarios (en el sentido alemán del término), el análisis de casos, los grupos de trabajo, la realización de proyectos, el trabajo en comunidades, la interacción con las empresas, etc. Los grupo de trabajo en seminarios y proyectos pueden posibilitar la acción interdisciplinaria y beneficiarse de un apto mecanismo para la integración de conocimientos. Escenarios como estos últimos constituyen un medio excelente para aprender a discutir sin pelear, comunicar con efectividad y hacer presentaciones. Otros objetivos pueden lograrse mediante un ambiente cultural en el campus universitario, las actividades extracurriculares, los clubes, centros, etc.

Dada la velocidad del cambio tecnológico y de la obsolescencia profesional, es indispensable un énfasis en la formación básica, al igual que en la flexibilidad curricular. Pero el Maestro Integrador ya mencionado no podrá obtenerse con la sola formación de un primer grado, pues aquel requiere la madurez y la acción investigadora que por lo general se adquieren después de estudios de posgrado. Es del caso pensar que en el mediano o largo plazo el ejercicio de la ingeniería exija un grado de Magíster, con énfasis en lo profesional, después de un primer grado en ciencias o en ciencias de la ingeniería.

Otra urgente tarea concomitante no puede ser eludida por las Facultades de Ingeniería. El uso de Internet está modificando tanto la docencia como el ejercicio profesional de la ingeniería, y Colombia enfrenta en este campo un retraso considerable. Grandes centros como la Universidad de Stanford y el Instituto Tecnológico de Georgia, en Estados Unidos, tienen ya estudios de maestría en ingeniería diseñados específicamente para la red mundial, y en España se anunció que a partir de septiembre entrará a funcionar la primera universidad virtual en castellano, la cual aspira a conseguir unos 40.000 estudiantes de España y América Latina para el año 2004 (4).

El uso de Internet y de los multimedios en el computador puede relevar al profesor de la tarea rutinaria de proporcionar información básica y permitirle entonces dedicar más tiempo a la comunicación del conocimiento tácito, el encuentro interpersonal con el estudiante, el trabajo con pequeños grupos, y la labor de síntesis, crítica y evaluación. La disminución del trabajo presencial puede permitir a las mejores universidades del país expandir virtualmente el campus, de modo que se revierta una tendencia actual: la gran demanda de cupos en la educación superior ha venido siendo atendida por universidades de menor desarrollo relativo o definitivamente mediocres.

\section{LA UNIVERSIDAD DEL FUTURO}

Para finalizar, el autor de estas líneas desea referirse a lo que cree será la universidad del futuro, algo que por supuesto tiene enorme importancia para la profesión de ingeniería. La universidad del futuro será aquella que logre el encuentro de científicos y técnicos, por un lado, con humanistas y artistas, por el otro. Dichas visiones, a pesar de ser complementarias y exigir interacción, se encuentran muy separadas, y ello puede explicar en algún grado una de las tragedias de los tiempos actuales: el avance y dominio de la tecnología, frente al retraso de los valores yla condición humana.

Recuérdese lo que se decía sobre el ingeniero como orientador de la tecnología. Si a la formación en este campo se agrega una mayor comprensión de la cultura, sensibilidad social y sentido de la solidaridad, se tendrá un profesional con una preparación excepcional para no sólo manejar la tecnología sino para 
orientarla hacia la consecución de fines nobles.

Aquí habría que hablar, más que de interdisciplinariedad, de conocimiento transdisciplinario, o sea, de un conocimiento que vaya más allá de una simple disciplina. Dice Edgar Morin en el prólogo a un documento de la UNESCO sobre la educación del futuro (5): "El humano es a la vez físico, biológico, síquico, cultural, social, histórico. Es esta unidad compleja la que está completamente desintegrada en la educación a través de las disciplinas y que imposibilita aprender lo que significa ser humano. Hay que restaurar dicha unidad compleja de tal manera que cada uno desde donde esté tome conciencia de su identidad compleja y de su identidad común".

Continúa Morin: "A partir de las disciplinas actuales, es posible reconocer la unidad y complejidad humanas mediante la organización de conocimientos dispersos en las ciencias de la naturaleza, en las humanas, en la literatura y la filosofía, y mostrar la unión indisoluble entre unidad y diversidad de todo lo humano".

\section{BIBLIOGRAFÍA}

(1) DUQUE, MAURICIO, et al, Formación de ingenieros para la innovación y el desarrollo tecnológico en Colombia, revista Dyna, Número 128, Medellín, 1999.

(2) Seminario Ingeniería, Investigación y Sociedad, Memorias, Colciencias, Universidad de Antioquia, Centro de Ciencia y Tecnología de Antioquia y Quirama, Medellín, 1998.

(3) http://web.mit.edu/civenv/

(4) La Universidad Oberta y Planeta anuncian la primera universidad virtual en castellano, El País, Madrid, 23 de junio de 2000.

(5) MORIN, EDGAR, Siete Saberes, prólogo a un documento de la Unesco, Lecturas Dominicales, El Tiempo, Bogotá, 13 de agosto de 2000. 